\title{
Atypical Polypoid Adenomyoma (APAM) of the Uterine: Relationship with Endometrial Cancer
}

\author{
Iori Kisu, Kouji Banno*, Megumi Yanokura, Yusuke Kobayashi, Arisa Ueki, Asuka Ono, \\ Kenta Masuda, Wataru Yamagami, Hiroyuki Nomura, Akira Hirasawa, Nobuyuki Susumu, \\ Daisuke Aoki
}

Department of Obstetrics and Gynecology, Keio University School of Medicine, Tokyo, Japan.

Email: *kbanno@sc.itc.keio.ac.jp

Received June $8^{\text {th }}, 2011$; revised June $30^{\text {th }}, 2011$; accepted August $8^{\text {th }}, 2011$.

\begin{abstract}
Atypical polypoid adenomyoma (APAM) is a rare polypoid tumor that generally occurs in women of reproductive age who have abnormal genital bleeding. The tumor was reported as a new disease concept by Mazur in 1981. Pathologically, APAM consist of irregularly proliferated endometrial gland cells and interlacing bundles of smooth muscle cells within the stroma, and have a similar form to adenocarcinoma, adenofibroma, adenosarcoma, and carcinosarcoma. Therefore, differential diagnosis is required in many cases. APAM is pathologically classified as a benign lesion and clinically has a comparatively favorable outcome. However, treatment and follow-up observation should be performed carefully because recurrence and residual lesions occur in many patients after conservative treatment. Concomitant development of endometrial adenocarcinoma also occurs in many cases and it is difficult to differentiate this disease from APAM. Thus, diagnosis of APAM should be made carefully, particularly since the number of cases of endometrial adenocarcinoma has increased in recent years. Furthermore, APAM tends to develop during a woman's reproductive years, and fertility sparing treatment should be considered. Here, we review the clinicopathological characteristics of APAM, including the difficulty of diagnosis as a benign or malignant uterine tumor, and we examine the relationship between APAM and endometrial cancer.
\end{abstract}

Keywords: Atypical Polypoid Adenomyoma, Endometrial Adenocarcinoma, Atypical Endometrial Hyperplasia, Transcervical Resectoscope, Fertility Sparing

\section{Introduction}

Atypical polypoid adenomyoma (APAM) was first reported by Mazur in 1981 as a tumor that mainly consisted of proliferated endometrial glands and smooth muscle cells [1]. Clinically, APAM is a rare sessile polypoid tumor that occurs in the uterus of premenopausal women manifests with abnormal genital bleeding, menstruation disorder, and hypermenorrhea.

APAM have an epithelial component consisting of endometrial glands with complex branches of cellular and structural atypia. In some cases, it is difficult to differentiate APAM from atypical endometrial hyperplasia (AEH). The stroma mainly consists of smooth muscle tissues, including various ratios of fibroblasts. Tumors requiring pathological differential diagnosis from APAM include adenocarcinoma, adenofibroma, adenosarcoma, and carcinosarcoma. Since the stroma in APAM consists of smooth muscle, differential diagnosis from well-dif- ferentiated endometrioid adenocarcinoma based on the samples by dilatation and curettage is difficult, and a method to avoid erroneous diagnosis is urgently required.

It is unclear whether APAM is a precancerous lesion of endometrioid adenocarcinoma. Appropriate treatment and follow-up of APAM patients are required for this reason and because residual lesions and recurrence can occur after conservative treatment. APAM also tends to develop during a woman's reproductive years, and consideration of fertility sparing treatment is important. The significant increase in endometrial cancer in recent years suggests the need for increased awareness of APAM among gynecologic oncologists.

\section{Definition and Clinical Presentation of APAM}

APAM develops in the fundus, lower part, and cervix of 
the uterus as a polypoid tumor that shows extroversive development on transvaginal ultrasonography, MRI, CT, PET-CT, and hysteroscopy. Differential diagnosis of APAM is required from endometrial polyp, submucosal myoma, and uterine body cancer. Diagnosis of APAM is straightforward when observed as a polypoid lesion from the external cervical os. In T2-weighted MRI, both high and low intensity areas are observed. In PET, accumulation of ${ }^{18} \mathrm{~F}$-FDP is generally viewed as a marker of a malignant tumor, but moderate accumulation may also occur in benign diseases such as leiomyoma. Such accumulation in benign diseases is related to the active proliferation potential of the tumor cells, and thus APAM should be suspected in a case with a polypoid lesion in the uterus for which accumulation differs from that in normal uterine myoma [2]. Visually, APAM appears as single or multiple polypoid nodular lesions with smooth yellow to ash gray surfaces. The size varies from $\leq 1 \mathrm{~cm}$ to several centimeters, with lesions of $\leq 2 \mathrm{~cm}$ being most common.

At present, APAM is histopathologically classified as a benign lesion of mixed epithelial and mesenchymal tumors (Table 1) [3] with a clear border with normal tissues and no invasive proliferation. APAM consist of irregularly proliferated endometrial glands and interlacing bundles of smooth muscle cells. The endometrial gland shows various levels of structural and cellular atypia, with a multilayered glandular epithelium, cribriform pattern, solid lesion, irregular branching, and papillary arrangements. Squamous metaplasia and squamous morule are found in about $90 \%$ of APAM cases, accompanied by elimination of the gland structure or necrosis in some cases [4-6]. Advanced cellular atypia such as polygonal cells, a large nucleus, and a large nucleolus are also observed in some cases. Longacre et al. [7] described images with partly similar characteristics to those of the structure of well-differentiated glandular cancer in

Table 1. Classification of mixed epithelial and mesenchymal tumors.

\begin{tabular}{c}
\hline Benign mixed epithelial and mesenchymal tumors \\
Adenofibroma \\
Adenomyoma \\
atypical polypoid adenomyoma \\
Malignant mixed epithelial and mesenchymal tumors \\
Adenosarcoma \\
homologous \\
heterologous \\
Carcinofibroma \\
Carcinosarcoma \\
homologous \\
heterologous
\end{tabular}

$45 \%$ of APAM cases, which were referred to as "APAMs with low malignant potential." Complications of welldifferentiated endometrioid adenocarcinoma and invasion in the muscle layer are also observed in some cases [8].

The stroma consists of smooth muscle cells that show bundle proliferation with less atypia, and myofibroblasts (intermediates between smooth muscle cells and fibroblasts) [4,7]. Although atypia of these stromal cells is seen in some APAM cases, the level of atypia is not high. Mitosis is also observed, but 2 or more mitoses in 10 fields of a high-power microscope have not been found [4]. The smooth muscle component of the stroma may also be incorrectly identified as muscle invasion of endometrioid adenocarcinoma, and care is required in interpreting the results. The cytoplasm is smaller than that of smooth muscle cells in the normal muscle layer, and polarity is lost in many cases. The endometrium of the non-tumor area shows high estrogen activity [9].

Cytological finding shows hyperplasia of the endometrium and a cluster of smooth muscle cells with less atypia. The latter finding is important for diagnosis, but a comprehensive perspective is required based on clinical presentation and images. Since concomitant development of endometrial adenocarcinoma can occur in many cases, a diagnosis based only on cytological finding should be made carefully [10]. A DNA ploidy test shows that all cases are DNA diploid and that the S-phase fraction is low [11].

APAM tends to develop in comparatively young premenopausal women, and especially in nulligravid women, with many cases having infertility. The average age at which APAM is diagnosed is about 40 years old [4], but the lesion may develop in postmenopausal women in rare cases. Abnormal genital bleeding is the chief complaint in most cases, and menstruation disorder and hypermenorrhea may also occur. Diagnosis should include data for menstrual history, pregnancy history, delivery history, history of hormone therapy, obesity, hypertension, diabetes, ovulation disorder, and infertility $[7,12$, 13].

Concomitant development of APAM has been reported in a patient with Turner's syndrome who was receiving long-term administration of an estrogenic drug [14]. This suggests a relationship between long-term stimulation by estrogen and a neoplasm in the uterine epithelium or stroma. Hyperprolactinemia may also cause amenorrhea, anovulation and infertility, and development of APAM has been reported after long-term continuous estrogen stimulation of precursor cells of the endometrial stroma in a woman with ovulation disorder [15]. Development of APAM may be affected by estrogen because the disease often develops in premenopausal and nul- 
ligravid women, and the endometrium shows endometrial hyperplasia or proliferation.

\section{Developmental Mechanism of APAM}

Many aspects of the histogenesis of APAM are unclear. Similarities of endometrial stromal cells in APAM and those in the endometrium or endocervix suggest that endometrial or related stromal cells in the endocervix are precursors of the smooth muscle component of APAM [11]. The close correlation between gland constituents and smooth muscle components is caused by neometaplasia of endometrial stromal cells, and smooth muscle and endometrial stroma coexist as stromal components in many cases with a uterine tumor and endometriosis.

\section{Treatment of APAM}

Total hysterectomy is an option for treatment of APAM in cases in which the symptoms in postmenopausal women are not improved by polypectomy or by dilatation and curettage. In other cases, the treatment choice depends on age, desire for a future pregnancy, and symptoms. For a young patient with a strong desire for preservation of the uterus, fertility sparing treatment is required based on the premise of correct pathological diagnosis of APAM. Fertility sparing treatment includes dilatation and curettage, polypectomy, and transcervical resection (TCR) of tumor.

Dilatation and curettage is sometimes performed as conservative therapy, but the treatment has problems because it is performed as a blind procedure in the uterine cavity. This leads to the possibility of a false negative finding in pathological diagnosis due to a small lesion being missed, and the limited collection of samples also causes difficulty in differential diagnosis between APAM and endometrioid adenocarcinoma with accompanying invasion in muscle layers $[7,11,16]$. Longacre et al. found a residual lesion or recurrence in $45 \%$ of patients who underwent curettage or polypectomy [7].

The incidence of endometrial cancer after conservative treatment is unclear, but the clinical course is comparatively favorable. In an analysis of 136 cases, Heatley found a residual lesion or recurrence in 41 patients (30.1\%), and a complication of hyperplasia in $12(8.8 \%)$ and carcinoma in $12(8.8 \%)$ [17]. In 9 of the 12 patients with carcinoma, carcinoma was found in the APAM lesion or at its base, and in the other 3 patients carcinoma was in the neighboring endometrium. Thus, as mentioned above, APAM may be a precancerous lesion. Advanced age and long-term administration of estrogenic drugs are also attracting attention as risk factors for malignant transformation of APAM, but the details remain unclear.

It is extremely difficult to estimate the incidence of endometrial adenocarcinoma that develops from APAM in patients who receive conservative treatment, because it is very difficult to differentiate these cases from patients who develop APAM and carcinoma concomitantly. The rate of transition to carcinoma in patients with complex AEH is $45 \%$ and the rate of progression is $14 \%$. These data suggest that the rate of progression in APAM is high, but the risk of concomitant carcinoma is lower than that in complex AEH $[16,18,19]$. However, these data show that all cases of APAM cannot be defined as benign lesions and a detailed examination should be performed for individual patients [20].

Sardo et al. proposed TCR for treatment of APAM, using four steps of removal of the APAM lesion under direct vision, removal of the area surrounding the lesion, removal of muscle layers in the lower part of the lesion, and biopsy of several linings of the endometrium, followed by pathological examination of resected samples [12]. This surgical method permits intracervical and intrauterine observation and biopsy of the lesion and the surrounding area, which allows more information to be obtained.

The above findings show that patients with APAM have high risks of residual lesions and recurrence, as well as concomitant development of carcinoma. Thus, in fertility sparing treatment, it is important that sufficient removal of muscle layers including the lesion should be performed under direct hysteroscopic vision [16]. Careful follow-up should then be performed, including targeted tissue biopsy using hysteroscopy, with consideration of the risk of malignant transformation and concomitant development of carcinoma.

\section{Endometrial Cancer and APAM}

In diagnosis, APAM should be differentiated from endometrioid adenocarcinoma, adenofibroma, adenosarcoma, and carcinosarcoma with invasion in muscle layers. These four diseases develop in postmenopausal women with a chief complaint of abnormal genital bleeding in many cases; while APAM tends to occur in younger women, but also with abnormal genital bleeding. Thus, age is an important factor in differential diagnosis (Table 2) [21].

APAM is visualized as a solid and polypoid lesion with a clear border. In contrast to many malignant tumors, no bleeding or necrotic focus is seen in APAM, whereas these symptoms are common in most adenosarcomas and carcinosarcomas $[4,7,11]$. The smooth muscle component of the stroma is increased in APAM compared to the normal uterine musculature and has characteristics that differ from those of the smooth muscle component of the stroma in adenofibroma and carcinosarcoma. The stroma in many cases of adenofibroma and carcinosarcoma is similar to the endometrial stroma, or 
Table 2. Clinicopathologic features of mixed epithelial stromal tumors of the uterus.

\begin{tabular}{|c|c|c|c|c|}
\hline & Adenofibroma & Atypical Polypoid Adenomyoma & Adenosarcoma & Carcinosarcoma \\
\hline Epithelial component & Benign glands & Crowded atypical glands & Benign glands & $\begin{array}{l}\text { Malignant (endometrioid, } \\
\text { serous, clear cell carcinoma) }\end{array}$ \\
\hline Stromal component & Benign fibroblastic & Smooth muscle & Malignant endometrial stroma & $\begin{array}{l}\text { High-grade sarcoma (ho- } \\
\text { mologous or heterologous) }\end{array}$ \\
\hline Age & Postmenopausal & Premenopausal & Varies & Postmenopausal \\
\hline
\end{tabular}

fibriform smooth muscle tissues are observed locally, although these findings may not always be clear. The stroma in adenofibroma is generally more atypical than that in APAM and it is rare that the smooth muscle component is clearly observed in adenofibroma and carcinosarcoma. In addition, gland tissues of the epithelial component show a papillary arrangement, cysts, and mild cellular atypism in many cases of adenofibroma, while severe cellular atypism is observed in both epithelial and stromal constituents as characteristics of carcinosarcoma.

Differentiation of APAM from endometrioid adenocarcinoma based on endometrium biopsy and curettage material is particularly important. Many cases of APAM may have been diagnosed as endometrioid adenocarcinoma before the description of APAM by Mazur. Even today, incorrect diagnosis of endometrioid adenocarcinoma may be made in many cases [22]. The gland in endometrioid adenocarcinoma histologically shows clear malignancy (solid, cribriform pattern, polymegethism of nuclei, large nuclei). No stroma is present in the proliferated gland duct or necrosis is apparent. APAM should always be suspected when interlacing bundles of smooth muscle cells is observed around a proliferated endometrial gland, with consideration of the age of the patient, images, and history of infertility. Care should also be taken since the stromal components in APAM and endometrial cancer both consist of myofibroblasts.

It is extremely important to differentiate APAM from muscle invasion of endometrioid adenocarcinoma. For this differentiation, interstitial reactions in the area of invasion, inflammatory cell infiltration, and granulomatous changes are observed in many cases. In APAM, smooth muscle cells are present in proximity to many ducts of the gland. A localized focal necrosis is sometimes observed in APAM, but the probability of endometrioid adenocarcinoma is higher in cases with necrosis over a comparatively large area. In endometrioid adenocarcinoma that develops concomitantly with adenomyosis, atypism of the gland is high, the stroma around the proliferated gland duct consists of endometrial stromal cells, and normal smooth muscle tissues with no atypia are observed around the stroma.
Differentiation of APAM from endometrioid adenocarcinoma Grade 1 with less cellular atypia is particularly difficult. Soslow et al. [9] reported that the immunophenotype of APAM did not differ significantly from that of well-differentiated endometrial adenocarcinoma and that immunophenotyping was of little value in distinguishing APAM from carcinoma. Nakamura et al. [23] suggested that a smooth muscle-like spindle cell stroma, a branched/cancellous vascular stroma, and a clear background are significant findings with high sensitivity/specificity for differentiation of APAM from endometrioid adenocarcinoma Grade 1 in cytological diagnosis of the endometrium. In addition, Ohishi et al. [24] proposed differentiation with CD10 immunostaining, since endometrial cancer accompanying invasion in muscle layers is positive for CD10, while APAM is negative or extremely mildly positive.

\section{Conclusions}

APAM is a new disease that was first reported only 30 years ago. The disease is rare and difficult to differentiate from endometrial cancer, and thus accumulation of cases and research data is currently insufficient. This is particularly true regarding pregnancy after fertility sparing treatment. Several case reports are available [25], but there are no detailed data on abortion rate, mode of delivery, and conditions of newborns. Data accumulation on a worldwide scale is required to address these questions, but this requires establishment of a simple and accurate method for differential diagnosis. Current research may help to clarify the mechanism of development of APAM and the relationship with estrogen. Research into risk factors for concomitant development of carcinoma and malignant transformation of APAM is also required to establish indications for fertility sparing treatment.

\section{REFERENCES}

[1] M. T. Mazur, "Atypical Polypoid Adenomyoma of the Endometrium," American Journal of Surgical Pathology, Vol. 5, No. 5, 1981, pp. 473-482.

[2] T. Maeda, U. Tateishi, Y. Sasajima, T. Hasegawa, H. Daisaki, Y. Arai and K. Sugimura, “Atypical Polypoid 
Adenomyoma of the Uterus: Appearance on 18F-FDG PET/MRI Fused Images," American Journal of Roentgenology, Vol. 186, No. 2, 2006, pp. 320-323. doi:10.2214/AJR.04.1799

[3] F. A. Tavassoli, "World Health Organization Classification of Tumors, Pathology and Genetics of Tumors of the Breast and Female Genital Organs," IARC Press, Lyon, 2003.

[4] R. H. Young, T. Treger and R. E. Scully, "Atypical Polypoid Adenomyoma of the Uterus; a Report of 27 Cases," American Journal of Clinical Pathology, Vol. 86, No. 2, 1986, pp. 139-145.

[5] D. C. Chhieng, P. A. Elgert, J. F. Cangiarella and J. M. Cohen, "Cytologic of Polypoid Adenomyomas: A Report of Two Cases," Diagnostic Cytopathology, Vol. 22, No. 3, 2000, pp. 176-180.

doi:10.1002/(SICI)1097-0339(20000301)22:3<176::AIDDC9>3.0.CO;2-H

[6] J. Kimura, H. Okamoto, H. Yamamoto, T. Mazaki and T. Kitamura, "Cytologic Futures of Atypical Polypoid Adenomyoma of the Endometrium-A Case Report," Acta Cytologica, Vol. 47, No.2, 2003, pp. 287-292.

[7] T. A. Longacre, M. H. Chung, R. V. Rouse and M. R. Hendrickson, "Atypical Polypoid Adenomyomas (Atypical Polypoid Adenomyomas) of the Uterus: A Clinicopathologic Study of 55 Cases," American Journal of Surgical Pathology, Vol. 20, No. 12, 1996, pp. 1-20.

[8] T. Sugiyama, S. Ota, T. Nishida, N. Okura, K. Tanabe and M. Yakushiji, "Two Cases of Endometrial Adenocarcinoma Arising from Atypical Polypoid Adenomyoma," Gynecologic Oncology, Vol. 71, No. 1, 1998, pp. 141-144. doi:10.1006/gyno.1998.5137

[9] R. A. Soslow, M. H. Chung, R. V. Rouse, M. R. Hendrickson and T. A. Longacre, "Atypical Polypoid Adenomyoma (APA) versus Well-Differentiated Endometrial Carcinoma with Prominent Stromal Matrix: An Immunohistochemical Study," International Journal of Gynecological Pathology, Vol. 15, No. 3, 1996, pp. 209-216.

[10] D. Baschinsky, S. Keyhani-Rofagha and A. Hameed, "Exfoliative Cytology of Atypical Polypoid Adenomyoma, a Case Report," Acta Cytologica, Vol. 43, No. 4, 1999, pp. 637-640.

[11] M. Fukunaga, Y. Endo, S. Ushigome and E. Ishikawa, "Atypical Polypoid Adenomyomas of the Uterus," Histopathology, Vol. 27, No. 1, 1995, pp. 35-42.

[12] A. Di Spiezio Sardo, I. Mazzon, V. Gargano, et al., "Hysteroscopic Treatment of Atypical Polypoid Adenomyoma Diagnosed Incidentally in a Young Infertile Woman," Fertility and Sterility, Vol. 89, No. 2, 2008, pp. 456.e9-e12. doi:10.1016/i.fertnstert.2007.02.061

[13] A. Y. Wong, K. S. Chan, W. L. Lau and L. C. Tang, "Pregnancy Outcome of a Patient with Atypical Polypoid Adenomyoma," Fertility and Sterility, Vol. 88, No. 5, 2007, pp. e7-e9. doi:10.1016/j.fertnstert.2007.01.043

[14] P. B. Clement and R. H. Young, "Atypical Polypoid Adenomyoma of the Uterus Associated with Turner's Syn- drome: A Report of Three Cases, Including a Review of 'Estrogen-Associated' Endometrial Neoplasms and Neoplasms Associated with Turner's Syndrome," International Journal of Gynecological Pathology, Vol. 6, No. 2, 1987, pp. 104-113.

[15] K. Nasu, T. Miyazaki, N. Takai and I. Miyakawa, "Atypical Polypoid Adenomyoma in a Patient with Hyperprolactinemia," International Journal of Gynecological Cancer, Vol. 11, No. 4, 2001, pp. 326-328.

[16] G. A. Viols and H. C. Ettler, "Atypical Polypoid Adenomyoma and Hysteroscopic Endometrial Ablation," Journal of Obstetrics and Gynaecology Canada, Vol. 25, No. 9, 2003, pp. 760-762.

[17] M. K. Heatley, "Atypical Polypoid Adenomyoma: A Systemic Review of the English Literature," Histopathology, Vol. 48, No. 5, 2006, pp. 609-610. doi:10.1111/j.1365-2559.2005.02315.x

[18] J. P. Baak, E. C. Wisse-Brekelmans, J. C. Fleege, et al., "Assessment of the Risk on Endometrial Cancer in Hyperplasia, by Means of Orphological and Morphological Features," Pathology Research and Practice, Vol. 188, No. 7, 1992, pp. 856-859.

[19] R. J. Kurman, P. F. Kaminski and H. J. Norris, "The Behavior of Endometrila Hyperplasia: A Long-Term Study of 'Untreated' Hyperplasia in 170 Patients," Cancer, Vol. 56, No. 2, 1985, pp. 403-412.

[20] M. Fukuda, N. Sakurai, Y. Yamamoto, et al., "Case of Atypical Polypoid Adenomyoma that Possibly Underwent a Serial Progression from Endometrial Hyperplasia to Carcinoma," Journal of Obstetrics and Gynaecology Research, Vol. 37, No. 5, 2011, pp. 468-471. doi: 10.1111/j.1447-0756.2010.01365.x

[21] J. S. Berek and N. F. Hacker, "Practical Gynecologic Oncology", 4th Edition, Williams \& Wilkins, Philadelphia, 2005.

[22] T. Kaku, H. Yoshikawa, et al., "Conservative Therapy for Adenocarcinoma and Atypical Endometrial Hyperplasia of the Endometrium in Young Women: Central Pathologic Review and Treatment Outcome," Cancer Letters, Vol. 167, No. 1, 2001, pp. 39-48.

doi:10.1016/S0304-3835(01)00462-1

[23] Y. Nakamura, M. Kawamoto, M. Matsubara, et al., "Cytological Features of Atypical Polypoid Adenomyoma," Journal of the Japanese Society of Clinical Cytology, Vol. 49, No. 3, 2010, pp. 171-176.

[24] Y. Ohishi, T. Kaku, H. Kobayashi, et al., "CD10 Immunostaining Distinguishes Atypical Polypoid Adenomyofibroma (Atypical Polypoid Adenomyoma) from Endometrial Carcinoma Invading the Myometrium," Human Pathology, Vol. 39, No. 10, 2008, pp. 1446-1453. doi:10.1016/j.humpath.2008.02.006

[25] M. Guida, E. Greco, A. Di Spiezio Sardo, et al., "Successful Pregnancy after Four-Step Hysteroscopic Technique for the Treatment of Atypical Polypoid Adenomyoma," Fertility and Sterility, Vol. 89, No. 5, 2008, pp. 1283-1284. doi:10.1016/j.fertnstert.2008.01.093 